ORIGINAL

\title{
Persistence of pathogens in liquid pig manure processed in manure tanks and biodigesters
}

\author{
Persistencia de patógenos en porcinaza líquida procesada en \\ tanques estercoleros y biodigestores
}

\author{
Oscar Betancur H, ${ }^{1}$ Ph.D, Antonio Betancourt E, ${ }^{2}$ Ph.D, Julián Estrada A, ${ }^{3}$ Ph.D, \\ Francisco Henao U, ${ }^{3 *}$ Ph.D.
}

\begin{abstract}
${ }^{1}$ Elanco, Animal Health, Transversal 18 96-41, Bogotá D.C., Colombia ${ }^{2}$ Corpoica, 140 street No 26 - 93, tower 2, Apartment 202, Floridablanca, Santander ${ }^{3}$ Caldas University, Faculty of Agricultural Sciences, Doctorate in Agricultural Sciences, Headquarters 65 street No 26-10, Manizales, Colombia. *Correspondence: fhenao@ucaldas.edu.co.
\end{abstract}

Received: November 2014; Accepted: July 2015.

\begin{abstract}
Objective. To evaluate the persistence of virus, bacteria, mold, yeast and parasites in liquid pig manure, processed in biodigesters and manure tanks in the central-western part of Colombia. Materials and methods. A directed observational study analyzed descriptively was carried out in three pig farms located where the manure tanks were assembled and its biodigesters were used. A sampling of liquid pig manure was taken to assess the presence of 26 pathogens at the beginning of the study and another one at the end of the process in manure tanks and biodigesters. For the manure tank, a 250 liters tank was filled with fresh pig manure and was analyzed after three days of storage. The biodigesters were of continuous flow and its effluents were analyzed, according to the specific hydraulic retention times. The diagnostic techniques were those recommended specifically for each microorganism and were carried out in certified labs by the Colombian Animal Health authority. Results. Of the 26 pathogens that were investigated, 15 appeared in the fresh pig manure used in pig manure tanks and 12 in the one used in biodigestors. In manure tanks, Porcine Circovirus type 2 (PCV2), mold, yeast, Salmonella spp., Balantidium coli and Strongylids did not persist. In biodigesters, PCV2, yeast, Strongylids, B. coli and Strongyloides spp., did not persist. Conclusions. In both manure tanks and biodigesters, a variation could be seen in pathogen persistency, indicating that they act as transformation systems of pig manure for the removal of the latter, as long as the storage times are increased if the efficiency wants to be improved.
\end{abstract}

Key words: Bacteria, mould, parasite, virus, yeast (Source: $C A B$ ).

\section{RESUMEN}

Objetivo. Evaluar la persistencia de virus, bacterias, mohos, levaduras, y parásitos en porcinaza líquida, procesada en biodigestores y tanques estercoleros en el centro-occidente de Colombia. Materiales y Métodos. Se realizó un estudio de observación dirigida analizada descriptivamente en tres explotaciones porcinas, donde se montaron tanques estercoleros y se aprovecharon sus biodigestores. Se realizó un muestreo de porcinaza fresca para analizar la presencia de 26 patógenos al comienzo del estudio y otro al final del proceso de estercoleros y biodigestores; los muestreos se repitieron en dos momentos en las tres granjas. Para el estercolero, se llenó un tanque de 250 litros 
con porcinaza fresca y se analizó después de tres días de almacenamiento. Los biodigestores fueron de flujo continuo, y se analizaron sus efluentes, según los tiempos de retención hidráulica específicos. Las técnicas diagnósticas fueron las recomendadas específicamente para cada microorganismo y se ejecutaron en laboratorios certificados por la autoridad sanitaria colombiana. Resultados. De los 26 patógenos investigados se detectaron 15 en la porcinaza fresca usada en estercoleros y 12 en la utilizada en biodigestores. En tanques estercoleros Circovirus Porcino tipo 2 (PCV2), mohos, levaduras, Salmonella spp., Balantidium coli y estrongilidos no persistieron. En biodigestores PCV2, levaduras, estrongilidos, B. coli y Strongyloides spp., no persistieron. Conclusiones. Tanto en estercoleros como biodigestores se observó variación en la persistencia de agentes patógenos, indicando que funcionan como sistemas de transformación de la porcinaza para la remoción de éstos, siempre y cuando se aumenten los tiempos de almacenamiento si se quiere mejorar su eficiencia.

Palabras clave: Bacterias, levaduras, mohos, parásitos, virus (Fuente: $C A B$ ).

\section{INTRODUCTION}

Pork is the most consumed animal protein in the world and the demand continues to grow. Its share of the world market is $37.4 \%$, which makes pig farming a highly important agricultural activity in the world (1). According to the Colombian Association of Pig Farmers (2), in Colombia the domestic pork market reached 3,030,043 heads slaughtered in 2013 , which represents approximately 254,000 tons of meat and indicates a growth of $2.2 \%$ compared to 2012 . This growth is expected to continue in the coming years; per capita consumption in Colombia is $6.7 \mathrm{~kg}$, which shows great future business opportunities. However, the large amount of pig manure that is produced daily limits the expansion of this industry, since it is associated with pollution and transmission of pathogens. Therefore, it is imperative to adequately dispose of and process pig manure so that it goes from being a source of pollution and health problems to a source of income for the producer. Proper use of pig manure may lead to it being used as a strategic bio-fertilizer or as raw material for animal feed.

Pig manure consists of feces, urine, bedding waste, rinse water, secretions (nose, throat, vagina, uterus, mammary gland, etc.), skin, hair, blood residue, and food waste. Organisms present in this mixture have the potential to persist in products derived from processing pig manure (3). To reduce the risk of contamination, pig manure should be treated using methods that ensure safety.

Diverse biological, physical and chemical processes to control pathogens in pig manure have been reported in literature, including: silage, composting, drying, earthworm composting, and the use of biodigesters, generally preceded by storage in manure tanks. For proper operation, with the exception of manure tanks, it is necessary to separate solid and liquid fractions of pig manure to aid in eliminating pathogens (4).

\section{INTRODUCCIÓN}

La carne de cerdo es la proteína de origen animal que más se consume en el mundo y su demanda sigue creciendo, su participación en el mercado mundial es del $37.4 \%$ lo cual convierte a la porcicultura en una actividad pecuaria de gran importancia en el planeta (1). Según la Asociación Colombiana de Porcicultores (2), en Colombia la oferta de porcinos en el mercado interno alcanzó 3'030.043 de cabezas beneficiadas en el 2013, que representan aproximadamente 254.000 toneladas de carne, indicando un crecimiento de $2.2 \%$ en comparación al año 2012 , y se espera que siga su crecimiento en los próximos años; el consumo per cápita en Colombia es de 6.7 $\mathrm{Kg}$ lo cual muestra una gran oportunidad de negocio a futuro. Sin embargo, la gran cantidad de porcinaza que se produce a diario limita la expansión de la industria porcina, dado que se le asocia con problemas de contaminación y de transmisión de patógenos; surge, por tanto, el imperativo de hacer una adecuada disposición y procesamiento de la porcinaza para que de un generador de problemas de contaminación y salud se transforme en una fuente de ingresos para el productor. El adecuado uso de la porcinaza puede permitir su utilización estratégica como biofertilizante o como materia prima para la alimentación animal.

La porcinaza, se compone de heces, orina, residuos de cama, agua de lavado, secreciones (nariz, garganta, vagina, útero, glándula mamaria, etc.), descamación cutánea, pelos, residuos de alimento y sangre. Los organismos presentes en esta mezcla tienen el potencial de persistir en los productos obtenidos del procesamiento de la porcinaza (3). Para disminuir el riesgo de contaminación, la porcinaza debe ser tratada por algún método que garantice su inocuidad.

Se han reportado en la literatura diferentes procesos biológicos, físicos y químicos para el 
To process liquid pig manure in the pork industry in Colombia, it is common to use manure tanks and biodigesters. Manure tanks are designed to store manure (5); in the case of pig manure, the manure tank is used to store the complete mixture without prior separation. Normally storage time is three days, after which its contents are dumped in a fertilization field or an anaerobic fermentation system in a digester. So far, no formal reports have been done on the effectiveness of manure tanks in eliminating pathogens; Fongaro et al (6) reported the presence of Salmonella spp. and PCV2 in fresh liquid pig manure collected from a manure tank, without taking into consideration the time period it was retained in order to establish the capacity this system has to remove pathogens. The manure tank, therefore, is not strictly a mechanism to process pig manure.

It is important to clarify that in some farms tanks similar to manure tanks are used to store effluent from the biodigesters. Biodigesters are bio-reaction systems built to submit the liquid phase of agricultural waste, manure or industrial effluents to anaerobic fermentation in order to recover energy and materials (biogas, bio-fertilizer, and potentially useful substrates in various agricultural and agro-industrial processes) (7).

Notably, biodigesters also offer the possibility to control the spread of pathogens (8), main factors that influence pathogen reduction, which include: microbial competition, nutrient availability (9), temperature, $\mathrm{pH}$, concentration of free ammonia and volatile fatty acids, biodigester design, and hydraulic retention time (HRT) or average time that the raw material is kept within the digester (10). To ensure an adequate biodigestion process, it is important that pig manure be sufficiently homogenized, and HRT should be sufficient to prevent endogenous bacteria from being expelled at a higher rate than that of reproduction (11). In this regard, Massé et al (12) reported significant reductions in the concentration of total and fecal coliforms, Escherichia coli, Salmonella spp., Campylobacter spp. and Yersinia enterocolitica when HRT was used for 7 to 14 days. In this sense, the Chen et al group (13) found that the rate of pathogen removal becomes more efficient as HRT increases from 11 to 25 days.

This study was done to help clarify real health risks from pig manure by evaluating the persistence of virus, bacteria, mold, yeast, and parasites in liquid pig manure processed in biodigesters and manure tanks in central-western Colombia. control de agentes patógenos en porcinaza, entre ellos están: el ensilaje, el compostaje, el secado, el lombricompostaje, y el uso de biodigestores, regularmente precedido del almacenamiento en tanques denominados estercoleros. Para el adecuado funcionamiento de estos procedimientos, con la excepción de los estercoleros, se requiere la separación de las fracciones sólida y líquida de la porcinaza, a lo cual se le atribuyen efectos benéficos en la eliminación de patógenos (4).

Para el procesamiento de la fase líquida de la porcinaza en la industria porcícola colombiana es común acudir a los estercoleros y a los biodigestores. Un estercolero es un tanque diseñado para almacenar estiércol (5), en el caso particular de la porcinaza, el estercolero se utiliza para almacenar la mezcla completa sin previa separación; regularmente el tiempo de almacenamiento es de tres días, al cabo de los cuales se conduce su contenido a un campo de fertilización o a un sistema de fermentación anaeróbica en un biodigestor. Hasta el momento no se conocen reportes formales sobre la efectividad de los tanques estercoleros en la eliminación de patógenos, Fongaro et al (6) reportan la presencia de Salmonella spp., y PCV2 en porcinaza fresca líquida colectada desde un tanque estercolero, sin considerar tiempos de retención que establezcan la capacidad de este sistema en la remoción de patógenos. El estercolero, por tanto, no es estrictamente un mecanismo de procesamiento de la porcinaza.

Es importante aclarar que en algunas granjas se usan tanques similares a los estercoleros para almacenar el efluente de los biodigestores. Los biodigestores, son sistemas de biorreacción construidos para someter la fase líquida de los desechos agrícolas, estiércol o efluentes industriales, a fermentación anaeróbica con el propósito de recuperar energía y materiales (biogás, biofertilizante, y posibles sustratos útiles en diversos procesos agropecuarios $y$ agroindustriales) (7).

Cabe destacar que los biodigestores también ofrecen la posibilidad de controlar la diseminación de agentes patógenos (8), los principales factores que influyen en la reducción de patógenos por este medio, incluyen: competición microbiana, disponibilidad de nutrientes (9), temperatura, $\mathrm{pH}$, concentración de amonio libre y de ácidos grasos volátiles, diseño del biodigestor, y tiempo de retención hidráulica (TRH) o tiempo promedio que la materia prima se mantiene dentro del biodigestor (10). Es importante que para poder asegurar un adecuado proceso de biodigestión, es necesario que la porcinaza esté 


\section{MATERIALS AND METHODS}

Type of study. A descriptively analyzed directed observational study was conducted.

Study site and population. The study was conducted in three full-cycle pig farms in central western Colombia, on a farm located in Pereira at $4^{\circ} 50^{\prime} 34^{\prime \prime} \mathrm{N}$; $75^{\circ} 46^{\prime} 02^{\prime \prime} \mathrm{W}$, on 800 sows (large farm), at an altitude of 1261 meters, average temperature of $25-30^{\circ} \mathrm{C}$; another on 500 sows (median farm) in Villamaría at $5^{\circ} 01^{\prime} 46^{\prime \prime} \mathrm{N}$; $75^{\circ} 31^{\prime} 36^{\prime \prime} \mathrm{W}$, at 1850 meters, with an average temperature of $21-22^{\circ} \mathrm{C}$; and 280 sows (small farm) in Santa Rosa de Cabal, at 4०50'34" $\mathrm{N}$; $75^{\circ} 46^{\prime} 02^{\prime \prime} \mathrm{W}$, at 1450 meters, average temperature 22 to $26^{\circ} \mathrm{C}$.

Treatment routes and sampling days. In 2013 fresh pig manure was sampled at baseline (June 4) and again when each route was processed (06, 07 and June 11); sampling was repeated on three farms (July 29 for fresh pig manure and July 31, August 01 and 05 for processed pig manure). On each farm a 250 plastic liter container was used as a manure tank, which was filled with 250 liters of fresh pig manure $(38 \mathrm{~kg}$ solid and $212 \mathrm{~L}$ liquid parts) and was stored for three days. Continuous flow polyethylene tube biodigesters (14) were used in the study with the following HRT: 2.55 days on the small farm, 2.65 days on the median farm and 6.8 days on the large farm. Manure tanks were sampled at the end of the third day of storage and the biodigesters according to the respective HRT; sampling was conducted following the regulations of the Colombian Technical Standards NTC-ISO 5667-1 (15). Samples were transported in sterile sealable bags with $75 \mathrm{~g}$ and $150 \mathrm{~g}$ frozen Biothermics $₫$ gels according to manufacturer's indications to preserve it at $4-8^{\circ} \mathrm{C}$, and the samples arrived at the laboratory in a period of less than 24 hours.

Pathogens and diagnostic techniques. In fresh pig manure the presence of the following pathogens (Table 1 ) was analyzed:

All the diagnostic tests were done in laboratories backed by the Colombian Agricultural Institute (ICA).

Selection criteria. The inclusion of the above pathogens in this study was done using the following criteria: PRRS, PCV2, PPV, $L$. intracellularis, A. pleuroneumoniae, for its high prevalence and economic impact. PCV1 was considered the control of PCV2. A. suum, E. coli, L. monocytogenes, Salmonella spp., Giardia spp., Leptospira spp., S. aureus due to the zoonotic suficientemente homogenizada, y que el TRH, sea el necesario para evitar que las bacterias endógenas sean expulsadas a una tasa superior a la de su reproducción (11). Al respecto, Massé et al (12) reportan reducciones significativas en la concentración de coliformes totales y fecales, Escherichia coli, Salmonella spp., Campylobacter spp. y Yersinia enterocolitica cuando se usan TRH de 7 a 14 días. En este mismo sentido, el grupo de Chen et al (13), encontró que la tasa de eliminación de patógenos se hace más eficiente conforme se aumenta el TRH de 11 a 25 días.

El presente trabajo se realizó con el propósito de contribuir a esclarecer el verdadero riesgo sanitario de la porcinaza mediante la evaluación de la persistencia de virus, bacterias, mohos, levaduras, y parásitos en porcinaza líquida, procesada en biodigestores y tanques estercoleros en el centro-occidente de Colombia.

\section{MATERIALES Y MÉTODOS}

Tipo de estudio. Se realizó un estudio de observación dirigida analizada descriptivamente.

Sitio y población de estudio. Se realizó un estudio en tres explotaciones porcinas de ciclo completo del centro occidente de Colombia, una granja ubicada en Pereira a $4^{\circ} 50^{\prime} 34^{\prime \prime} \mathrm{N}$; $75^{\circ} 46^{\prime} 02^{\prime \prime} \mathrm{O}$, de 800 madres (granja grande), a una altitud de $1261 \mathrm{msnm}$, y con temperatura media de $25-30^{\circ} \mathrm{C}$; otra de 500 madres (granja mediana) en Villamaría a $5^{\circ} 01^{\prime} 46^{\prime \prime} \mathrm{N}$; $75^{\circ} 31^{\prime} 36^{\prime \prime}$ $\mathrm{O}$, a $1850 \mathrm{msnm}$, y con una temperatura media de $21-22^{\circ} \mathrm{C}$; y una de 280 madres (granja pequeña) en Santa Rosa de Cabal, a $4^{\circ} 50^{\prime} 34^{\prime \prime}$ $\mathrm{N}$; 7546'02" O, a $1450 \mathrm{msnm}$, y $22-26^{\circ} \mathrm{C}$ de temperatura media.

Rutas de tratamiento y días de muestreo. Durante el año 2013 se hizo un muestreo de porcinaza fresca al comienzo del estudio (04 de junio) y otro al final del proceso de cada ruta (06, 07 y 11 de junio); el muestreo se repitió en las tres granjas ( 29 de julio para la porcinaza fresca, y 31 de julio, 01 y 05 de agosto para la porcinaza procesada). En cada granja se usó un recipiente plástico de 250 litros de capacidad, como estercolero, que se llenó con 250 litros de porcinaza fresca (38 $\mathrm{kg}$ de la fracción sólida y $212 \mathrm{~L}$ de la fracción líquida) y se almacenó por tres días. En el estudio se usaron biodigestores tubulares de polietileno y de flujo continuo (14), con los siguientes TRH: 2.55 días en la granja pequeña, 2.65 días en la mediana y 6.8 días en la granja grande. Los estercoleros se muestrearon al final del tercer día de almacenamiento y los biodigestores, de acuerdo con el TRH respectivo; 
Table 1. Studied microorganisms and diagnostic methods.

\begin{tabular}{|c|c|}
\hline Microorganism & Diagnostic methods \\
\hline $\begin{array}{l}\text { Porcine Parvovirus (PPV), } \\
\text { Porcine Circovirus type } \\
1 \text { (PCV1), PCV2, Porcine } \\
\text { Herpesvirus type } 1 \\
\text { (HVP1) or the Aujesky } \\
\text { disease virus }\end{array}$ & PCR multiplex (16) \\
\hline $\begin{array}{l}\text { Actinobacillus } \\
\text { pleuropneumoniae and } \\
\text { Lawsonia intracellularis }\end{array}$ & Conventional PCR $(17,18)$ \\
\hline $\begin{array}{l}\text { Porcine Reproductive } \\
\text { and Respiratory } \\
\text { Syndrome (PRRS) }\end{array}$ & Nested RT-PCR (19) \\
\hline $\begin{array}{l}\text { Clostridium sulfite } \\
\text { reducers }\end{array}$ & $\begin{array}{l}\text { TSN agar count (Sigma-Aldrich, St. Louis, } \\
\text { MO) }\end{array}$ \\
\hline $\begin{array}{l}\text { Aerobic mesophilic } \\
\text { bacteria }\end{array}$ & $\begin{array}{l}\text { Count in Petrifilm mesophilic plates ( } 3 \mathrm{M}^{\mathrm{TM}} \text {, } \\
\text { St. Paul, MN) with agar Plate Count (Merck- } \\
\text { Millipore, Billerica, MA) }\end{array}$ \\
\hline Staphylococcus aureus & $\begin{array}{l}\text { Count in Baird Parker culture medium with } \\
\left.\text { DNA ( } 3 \mathrm{M}^{\mathrm{TM}} \text {, St. Paul, } \mathrm{MN}\right) \text {, to determine the } \\
\text { production of DNAasa }\end{array}$ \\
\hline $\begin{array}{l}\text { Total coliforms and } E \text {. } \\
\text { coli }\end{array}$ & $\begin{array}{l}\text { Count in Bilis red Violet agar with } \\
\text { glucoronide }\left(3 \mathrm{M}^{\mathrm{TM}} \text {, St. Paul, } \mathrm{MN}\right)\end{array}$ \\
\hline Mold and yeast & $\begin{array}{l}\text { Count in mold and yeast Petrifilm plates } \\
\left(3 \mathrm{M}^{\mathrm{TM}} \text {, St. Paul, MN }\right)\end{array}$ \\
\hline Salmonella spp. & $\begin{array}{l}\text { Sequential incubation of } 25 \mathrm{~g} \text { sample } \\
\text { in lactose broth (3M } \mathrm{Tm} \text {, St. Paul, MN), } \\
\text { Rappaport broth (3M тм, St. Paul, MN), } \\
\text { Hektoen agar (Sigma-Aldrich, St. Louis, } \\
\text { MO), Bismuth sulphite agar (Merck- } \\
\text { Millipore, Billerica, MA) and Trypticase agar } \\
\text { (Merck-Millipore, Billerica, MA), Gram stain, } \\
\text { oxidase test (Merck-Millipore, Billerica, MA) } \\
\text { and agglutination with polyvalent antiserum } \\
\text { "O" (Prolab, Antioquia, Col) }\end{array}$ \\
\hline Listeria monocytogenes & $\begin{array}{l}\text { Incubation in } 25 \mathrm{~g} \text { of Fraser sample broth } \\
\text { (Merck-Millipore, Billerica, MA), and ELFA } \\
\text { test (Enzyme Linked Fluorescent Assay) } \\
\text { in miniVIDAS (BioMérieux, Craponne, Fr) }\end{array}$ \\
\hline Leptospira spp. & $\begin{array}{l}\text { Filtration with clarifying paper and } 0.45 \\
\text { micron cellulose acetate membrane, with } \\
\text { subsequent centrifugation and sediment } \\
\text { planting in EMJH medium (Ellinghausen } \\
\text { and McCullough as modified by Johnson } \\
\text { and Harris) (Difco TM, Detroit, MI) and } \\
\text { evaluated weekly for four weeks using } \\
\text { darkfield microscopy. }\end{array}$ \\
\hline $\begin{array}{l}\text { Ascaris suum, } \\
\text { Trichuris suis, B. } \\
\text { coli, Strongyloides } \\
\text { spp., strongylids, } \\
\text { Metastrongylus spp., } \\
\text { and coccidias }\end{array}$ & $\begin{array}{l}\text { McMaster Sloss. Coccidia are incubated } \\
\text { with a solution of potassium permanganate } \\
\text { at } 1 \% \text { for } 24-48 \text { hours, and then the } \\
\text { respective micrometer measurements are } \\
\text { performed on the sporulated oocysts, }\end{array}$ \\
\hline Giardia intestinalis & Ritchie \\
\hline Cryptosporidium parvum & Zielh-Neelsen cold stain \\
\hline
\end{tabular}

risk involved. The virus for Aujesky's disease because of the risk it represents despite not being officially reported in Colombia. Aerobic mesophilic bacteria, total coliforms, Clostridium sulfite reducers, molds and yeasts since over time they are indicators of microbial activity. The other bacteria and parasites ( $T$. suis, B. coli, G. intestinalis, Strongyloides spp., Metastrongylus spp., coccidia and strongylid) because of their economic impact, epidemiological importance and because they are potentially pathogenic to other species, including humans.

In this study the persistence of viruses, parasites, A. pleuropneumoniae, Leptospira spp., $L$. el muestreo se realizó siguiendo las pautas de la Norma Técnica Colombiana NTC-ISO 5667-1 (15). Las muestras se transportaron en bolsas estériles con cierre hermético, acompañadas de geles Biothermics ${ }^{\circ}$ de $75 \mathrm{~g}$ y $150 \mathrm{~g}$ congelados según indicaciones del fabricante para conservar el frío de $4-8^{\circ} \mathrm{C}$, y llegaron al laboratorio en un periodo inferior a 24 horas después de tomada la muestra.

Patógenos y técnicas diagnósticas. En la porcinaza fresca se analizó la presencia de los siguientes patógenos (Tabla 1):

Todas las pruebas diagnósticas se ejecutaron en laboratorios avalados por el Instituto Colombiano Agropecuario (ICA).

Criterios de selección. La inclusión de los patógenos anteriores en esta investigación se hizo bajo los siguientes criterios: PRRS, PCV2, PVP, L. intracellularis, A. pleuroneumoniae, por su prevalencia y alto impacto económico. El PCV1 se consideró como control del PCV2. A. suum, E. coli, L. monocitogenes, Salmonella spp., Giardia spp., Leptospira spp., S. aureus por el riesgo zoonótico que conllevan. El virus de la enfermedad de Aujesky por el riesgo que esta representa a pesar de no estar reportado oficialmente en Colombia. Mesófilos aerobios, coliformes totales, Clostridium sulfito reductores, mohos y levaduras por ser indicadores de actividad microbiana en el tiempo. El resto de bacterias y parásitos ( $T$. suis, B. coli, G. intestinalis, Strongyloides spp., Metastrongylus spp., coccidias y estrongilidos) por su impacto económico, su importancia epidemiológica y por ser potencialmente patógenas para otras especies, incluidos los humanos.

En el presente estudio la persistencia de virus, parásitos, A. pleuroneumoniae, Leptospira spp., L. monocytogenes, L. intracellularis y Salmonella spp., se valoró a partir de la presencia del organismo, en tanto que para los organismos diagnosticados por recuento (mesófilos aerobios, S. aureus, coliformes totales, E. coli, Clostridium sulfito reductores, mohos, levaduras), la persistencia dependió de que el número final de cada agente superara los niveles máximos permitidos de acuerdo con los valores de referencia de la tabla 2 .

\section{RESULTADOS}

En la tabla 3 se aprecia que de los 26 patógenos estudiados, solo se encontraron 15 en la porcinaza fresca con la cual se llenaron los estercoleros; al tercer día de almacenamiento en esta vía se comprobó la persistencia de 9 de 
monocytogenes, L. intracellularis and Salmonella spp., was assessed from the presence of the organism, while for those diagnosed by counting (aerobic mesophilic bacteria, S. aureus, total coliforms, E. coli, Clostridium sulfite reducers, molds, yeasts), persistence depended on the final number of each agent in excess of the maximum permitted levels according to the reference values in table 2 .

\section{RESULTS}

Table 3 shows that of the 26 pathogens studied, only 15 were found in fresh pig manure which the manure tanks were filled with; after three days of storage 9 of them persisted ( $L$. intracellularis, mesophilic aerobic, E. coli, total coliforms, $S$. aureus, Clostridium sulfite reducers, $L$. monocytogenes, Strongyloides spp., coccidia); therefore, the other 6 disappeared during this period. E. coli, total coliforms, and Clostridium sulfite reducers persisted on the 3 farms; aerobic mesophilic bacteria persisted in the 3 farms but only in the largest it persisted in 2 samples; $S$. aureus persisted on the 3 farms, and did not

Table 3. Pathogens found in manure tanks on three farms in central western Colombia.

\begin{tabular}{|c|c|c|c|c|c|c|c|c|c|c|c|c|}
\hline \multirow{3}{*}{ PATHOGEN } & \multicolumn{4}{|c|}{ GG } & \multicolumn{4}{|c|}{ GM } & \multicolumn{4}{|c|}{ GP } \\
\hline & \multicolumn{2}{|c|}{ M1 } & \multicolumn{2}{|c|}{ M2 } & \multicolumn{2}{|c|}{ M1 } & \multicolumn{2}{|c|}{ M2 } & \multicolumn{2}{|c|}{ M1 } & \multicolumn{2}{|c|}{ M2 } \\
\hline & DI & $\mathbf{P}$ & DI & $\mathbf{P}$ & DI & $\mathbf{P}$ & DI & $\mathbf{P}$ & DI & $\mathbf{P}$ & DI & $\mathbf{P}$ \\
\hline \multicolumn{13}{|l|}{ PPV } \\
\hline \multicolumn{13}{|l|}{ PCV1 } \\
\hline PCV2 & & & & & & & + & & & & + & \\
\hline \multicolumn{13}{|l|}{ PRRS } \\
\hline \multicolumn{13}{|l|}{ HVP1 } \\
\hline L. intracellularis & + & + & + & + & + & & & & + & & + & + \\
\hline \multicolumn{13}{|l|}{ A. pleuropneumoniae } \\
\hline Aerobic mesophilic bacteria & + & + & + & + & + & & + & + & + & & + & + \\
\hline S. aureus & + & & + & + & & & + & + & & & + & + \\
\hline Mold & + & & & & + & & + & & & & & \\
\hline Yeast & + & & + & & + & & + & & & & + & \\
\hline $\begin{array}{l}\text { Clostridium sulfite } \\
\text { reducers }\end{array}$ & + & + & + & + & + & + & + & + & + & + & + & + \\
\hline Total coliform & + & + & + & + & + & + & + & + & + & + & + & + \\
\hline E. coli & + & + & + & + & + & + & + & + & + & + & + & + \\
\hline L. monocytogenes & & & & & & & & & & & + & + \\
\hline Salmonella spp. & & & & & + & & & & + & & & \\
\hline \multicolumn{13}{|l|}{ Leptospira spp. } \\
\hline \multicolumn{13}{|l|}{ A. suum } \\
\hline \multicolumn{13}{|l|}{ C. parvum } \\
\hline \multicolumn{13}{|l|}{ T. suis } \\
\hline B. coli & + & & & & & & + & & + & & + & \\
\hline \multicolumn{13}{|l|}{ Metastrongylus spp. } \\
\hline \multicolumn{13}{|l|}{ G. intestinalis } \\
\hline Strongylids & + & & & & + & & & & & & & \\
\hline Strongyloides spp. & + & & & & + & & + & + & & & + & \\
\hline Coccidia & + & + & + & + & + & + & + & + & + & + & + & \\
\hline
\end{tabular}

GG: large farm; GM: median farm; GP: small farm; M1: sampling 1; M2: sampling 2; DI: initial diagnosis (fresh pig manure); P: persistence; $(+)$ : presence of pathogen agent.
Table 2. Maximum counts adopted for ruminant feed.

\begin{tabular}{lcc}
\hline \multicolumn{1}{c}{ Microorganism } & $\begin{array}{c}\text { Maximum amount } \\
\text { CFU/g }\end{array}$ & Source \\
\hline Aerobic mesophilic bacteria & $10 \times 10^{7}$ & $(20)$ \\
S. aureus & $10 \times 10^{4}$ & $(21)$ \\
Molds & $10 \times 10^{4}$ & $(20)$ \\
Yeast & $10 \times 10^{4}$ & $(20)$ \\
Clostridium sulfite reducers & $20 \times 10^{1}$ & $(20)$ \\
Total coliforms & $10 \times 10^{4}$ & $(20)$ \\
E.coli & $10 \times 10^{1}$ & $(22)$ \\
\hline
\end{tabular}

CFU/g: Colony-forming units/gram

ellos (L. intracellularis, mesófilos aerobios, $E$. coli, coliformes totales, S. aureus, Clostridium sulfito reductores, $L$. monocytogenes, Strongyloides spp., coccidias), por tanto los otros 6 desaparecieron durante este período. E. coli, coliformes totales, Clostridium sulfito reductores, persistieron siempre en las 3 granjas; mesófilos aerobios persistieron en las 3 granjas pero solo en la grande persistieron en los 2 muestreos; $S$. aureus persistió en las 3 granjas, y en la grande no persistió en un muestreo; $L$. intracellularis persistió en la granja grande, en la mediana apareció solo en uno de los muestreos y no persistió y en la pequeña apareció en los 2 muestreos pero solo persistió en uno. Strongyloides spp., solo persistió en un muestreo de la granja mediana. Para el caso de coccidias estas persistieron en las 3 granjas, excepto en el segundo muestreo de la granja pequeña.

En los datos registrados en la tabla 4, se percibe que 12 de los 26 agentes incluidos en el estudio fueron diagnosticados inicialmente en la porcinaza líquida empleada para alimentar los biodigestores, 8 de ellos persistieron ( $L$. intracellularis, mesófilos aerobios, $S$. aureus, Clostridium sulfito reductores, coliformes totales, E. coli, Salmonella spp., coccidias). E. coli persistió siempre en las 3 granjas; L. intracellularis persistió en la granja pequeña, en la mediana apareció solo en uno de los muestreos y persistió y en la grande apareció en los 2 muestreos pero solo persistió en uno. Los mesófilos aerobios persistieron en las 3 granjas aunque solo en el primer muestreo. S. aureus persistió en la granja mediana y en la pequeña en uno de los muestreos, mientras en la granja grande no persistió. Para el caso de Salmonella spp., estas aparecieron solo en el primer muestreo de las 3 granjas y persistieron en la mediana y pequeña, en la grande no persistieron. Los Clostridium sulfito reductores persistieron en las granjas mediana y pequeña, y en la grande a pesar que apareció en los 2 muestreos solo persistió en uno de ellos. Se observaron coccidias en uno solo de los muestreos de las granjas grande y pequeña, y en ambos casos persistieron. 
Table 4. Pathogens found in biodigesters on three farms in central western Colombia.

\begin{tabular}{|c|c|c|c|c|c|c|c|c|c|c|c|c|}
\hline \multirow{3}{*}{ PATHOGEN } & \multicolumn{4}{|c|}{ GG } & \multicolumn{4}{|c|}{ GM } & \multicolumn{4}{|c|}{ GP } \\
\hline & \multicolumn{2}{|c|}{ M1 } & \multicolumn{2}{|c|}{ M2 } & \multicolumn{2}{|c|}{ M1 } & \multicolumn{2}{|c|}{ M2 } & \multicolumn{2}{|c|}{ M1 } & \multicolumn{2}{|c|}{ M2 } \\
\hline & DI & $\mathbf{P}$ & DI & $\mathbf{P}$ & DI & $\mathbf{P}$ & DI & $\mathbf{P}$ & DI & $\mathbf{P}$ & DI & $\mathbf{P}$ \\
\hline \multicolumn{13}{|l|}{ PPV } \\
\hline \multicolumn{13}{|l|}{ PCV1 } \\
\hline PCV2 & & & & & & & + & & & & + & \\
\hline \multicolumn{13}{|l|}{ PRRS } \\
\hline \multicolumn{13}{|l|}{ HVP1 } \\
\hline L. intracellularis & + & + & + & & + & + & & & + & + & + & + \\
\hline \multicolumn{13}{|l|}{ A. pleuropneumoniae } \\
\hline Aerobic mesophilic bacteria & + & + & + & & + & + & + & & + & + & + & \\
\hline S. aureus & + & & + & & + & & + & + & + & & + & + \\
\hline \multicolumn{13}{|l|}{ Mold } \\
\hline Yeast & + & & + & & + & & & & + & & & \\
\hline Clostridium sulfite reducers & + & + & + & & + & + & + & + & + & + & + & + \\
\hline Total coliform & + & & + & & + & + & + & + & + & + & + & \\
\hline E. coli & + & + & + & + & + & + & + & + & + & + & + & + \\
\hline \multicolumn{13}{|l|}{ L. monocytogenes } \\
\hline Salmonella spp. & + & & & & + & + & & & + & + & & \\
\hline \multicolumn{13}{|l|}{ Leptospira spp. } \\
\hline \multicolumn{13}{|l|}{ A. suum } \\
\hline \multicolumn{13}{|l|}{ C. parvum } \\
\hline \multicolumn{13}{|l|}{ T. suis } \\
\hline \multicolumn{13}{|l|}{ B. coli } \\
\hline \multicolumn{13}{|l|}{ Metastrongylus spp. } \\
\hline \multicolumn{13}{|l|}{ G. intestinalis } \\
\hline Strongylids & + & & & & & & & & & & & \\
\hline Strongyloides spp. & & & & & + & & & & & & + & \\
\hline Coccidia & & & + & + & & & & & & & + & + \\
\hline
\end{tabular}

GG: large farm; GM: median farm; GP: small farm; M1: sampling 1; M2: sampling 2; DI: initial diagnosis (fresh pig manure); P: persistence; (+): presence of pathogen agent.

persist in one sampling of the large farm; $L$. intracellularis persisted in the largest farm, in the median one it appeared in only one of the samples and did not persist, and in the small one it appeared in two samples but only persisted in one. Strongyloides spp., persisted only in a sampling of the median farm. Coccidia persisted on the 3 farms, except in the second sampling of the small farm.

In data recorded in table 4 , it can be seen that 12 of the 26 agents included in the study were initially diagnosed in liquid pig manure used to feed biodigesters, and 8 persisted ( $L$. intracellularis, aerobic mesophilic bacteria, S. aureus, Clostridium sulfite reducers, total coliforms, E. coli, Salmonella spp., coccidia). E. coli persisted in the 3 farms; $L$. intracellularis persisted in the small farm, appeared in the median one in only one of the samples and persisted, and appeared in the large farm in the 2 samples but persisted in just one. Aerobic mesophilic bacteria persisted in the 3 farms but only in the first sampling. S. aureus persisted in median and on the small farm in one of the

\section{DISCUSIÓN}

A la porcinaza se le atribuye un papel protagónico en la transmisión de agentes patógenos diversos (virus, bacterias, mohos, levaduras y parásitos), por lo cual se limita considerablemente su uso en procesos agropecuarios y agroindustriales. Por experiencias previas se sabe que ciertos agentes etiológicos pueden ser removidos total o parcialmente en el procesamiento de la porcinaza, mediante el uso de estercoleros, biodigestores, secado al sol, ensilaje, lombricompostaje y elaboración de harina de lombriz. El uso estratégico de la porcinaza, bien en biofertilización bien en alimentación animal, podría hacerse antecedido de procesos similares a los mencionados, y su verdadero rol en la transmisión de patógenos estaría determinado por la persistencia de los mismos al final de cada uno, o dicho de otra manera por el poder de remoción de estos procedimientos.

De los 26 patógenos inicialmente investigados aparecieron 15 en la porcinaza fresca usada para los estercoleros y 12 en la utilizada en 
samples, while in the large farm it did not persist. In the case of Salmonella spp., these appeared only with the first sampling of the 3 farms and persisted in the median and small one, but in the large farm it did not persist. Clostridium sulfite reducers persisted in the medium and small farms, and the large one, in spite of appearing in the 2 samples, persisted in just one. Coccidia was observed in just one of the samples of the large and small farms, and persisted in both cases.

\section{DISCUSSION}

Pig manure is considered to have a leading role in the transmission of various pathogens (virus, bacteria, molds, yeasts and parasites), so its use in agricultural and agro-industrial processes is considerably limited. From previous experience we know that certain etiological agents can be removed completely or partially by processing pig manure using tanks, biodigesters, sun-drying, silage, worm composting and by manufacturing worm flour. The strategic use of pig manure, either in biofertilization or in animal feed, could be possible if preceded by similar processes, and its true role in the transmission of pathogens would be determined by persistence in the end product, or in other words, if these procedures are able to remove them.

Of the 26 pathogens initially investigated, 15 were found in fresh pig manure used in the manure tanks, and 12 in manure used in the biodigesters, perhaps because they were not present in the farms studied (negative farms), or due to differences in health and biosecurity conditions, or due to very low prevalence at the time of sampling. Also, the presence of antibiotic residues that modify the bacterial populations in pig manure should not be ruled out (23). Meanwhile, persistence could be due to short storage processing times, especially in the case of high amounts of pathogens. For this reason, some authors $(13,24)$ consider longer retention times than those used in this study to be necessary.

In the viruses evaluated in this research, only PCV2 was found in both fresh pig manure used for the process and biodigesters (Tables 3 and 4 ), and in both cases it was removed. This virus has been found by other authors in liquid pig manure and has persisted in this environment even after 30-40 days (6); these authors argue that PCV2, a DNA virus, is resistant to disinfection and is commonly excreted in pig feces and urine; for those reasons, they are considered bioindicators of environmental quality. Although in this study it was not possible to pinpoint what caused the removal of the viruses found in fresh biodigestores, debido quizá a que o no se presentan en las granjas estudiadas (granjas negativas), o a las diferencias en las condiciones sanitarias y de bioseguridad, o por una prevalencia muy baja en el momento del muestreo. Además, no debe descartarse la presencia de residuos de antibióticos que modifiquen las poblaciones bacterianas en la porcinaza (23). Por su parte la persistencia podría deberse a los bajos tiempos del proceso de almacenamiento, sobre todo frente a cargas de patógenos altas; en este sentido, algunos autores $(13,24)$ consideran tiempos de retención mayores a los desarrollados en este estudio.

De los virus evaluados en esta investigación sólo se encontró el PCV2 tanto en la porcinaza fresca usada para el proceso en estercoleros y biodigestores (Tablas 3 y 4), y en ambos casos fue removido. Este virus ha sido hallado por otros autores en porcinaza líquida y ha persistido en este medio aún después de 30 a 40 días (6); estos mismos autores aducen que PCV2, por ser un virus DNA es resistente a la desinfección y es comúnmente excretado en las heces y orina de los cerdos; por esas razones, son considerados bioindicadores de calidad ambiental. Aunque en este estudio no es posible precisar la causa de la remoción de los virus que se encontraron en la porcinaza fresca, existe evidencia que en la porcinaza líquida algunos compuestos proteolíticos o el ion amonio tienen efectos deletereos sobre éstos agentes (23).

En el caso de L. intracellularis, hubo persistencia tanto en los tanques estercoleros como en los biodigestores. A pesar de la importancia clínica y económica de $L$. intracellularis, no ha sido considerada en los estudios la eficiencia de biodigestores o estercoleros en el tratamiento de la porcinaza, posiblemente porque es un agente patógeno que no afecta a otras especies de interés pecuario, ni es zoonótica, o por las dificultades que se presentan para su cultivo e identificación. Últimamente, se ha recomendado su identificación por PCR; sin embargo, la utilización de esta técnica puede indicar un patrón erróneo en el valor de persistencia encontrado, dada su capacidad de utilizar DNA a partir de células muertas (13).

En este estudio se encontró persistencia de mesófilos aerobios tanto en los tanques estercoleros, como en los biodigestores. En el caso de los tanques estercoleros, podría explicarse por las condiciones ecológicas dentro de los tanques, puesto que durante el almacenamiento de porcinaza, se presentan condiciones aerobias en la superficie, pero después de $30 \mathrm{~cm}$ de profundidad, las condiciones anaerobias 
pig manure, there is evidence that in liquid pig manure there are some proteolytic or ammonium ion compounds that have deleterious effects on these agents (23).

In the case of $L$. intracellularis, there was persistence both in manure tanks and biodigesters. Despite the clinical and economic importance of $L$. intracellularis, its efficiency has not been considered in studies of biodigesters or manure tanks to treat pig manure, possibly because it is a pathogen that does not affect other species of agricultural interest, it is not zoonotic, or due to difficulties in cultivating and identifying it. Lately, identifying it by PCR has been recommended; however, this technique may indicate an erroneous pattern in the persistence value found, given their ability to use DNA from dead cells (13).

In this study the persistence of aerobic mesophilic bacteria was found both in manure tanks and biodigesters. For manure tanks, it could be explained by the environmental conditions within the tanks, since during pig manure storage aerobic conditions are present on the surface, but $30 \mathrm{~cm}$ from the surface anaerobic conditions prevail (25). Biodigesters in this study showed persistence in only one sample. Chen et al (13) mention that different HRT lead to modifying the structure of the microbial community, which can have an effect on the survival of pathogenic bacteria.

S. aureus persisted both in the manure tanks and biodigesters. This is a facultative anaerobic bacteria, and this could favor its survival in both systems. Although the ammonium present in the liquid excreta has a bactericidal effect, given the lower cell permeability of gram positive cocci it is less susceptible than other bacteria (26). According to Poudel et al (27), it is only possible to eliminate $S$. aureus after a HRT of 10 days, and this study reinforces this approach, since the longest HRT was 6.8 days.

As for molds and yeasts, persistence was not found in manure tanks or biodigesters. There are few data on yeast and mold counts in animal manure (4). Greater amounts of bacteria than mold has been found in pig manure treated by anaerobic digestion because bacteria has a greater role in converting organic waste during this process (28). This study demonstrates the efficiency of treatment systems for liquid pig manure to remove these agents, given its susceptibility to ammonia and humic substances (28). prevalecen (25). En el caso de los biodigestores de este trabajo, se presentó persistencia en solo una de las muestras. Chen et al (13), mencionan que diferentes TRH conducen a la modificación de la estructura de la comunidad microbiana, que puede tener un efecto en la supervivencia de las bacterias patógenas.

S. aureus persistió tanto en los tanques estercoleros como en los biodigestores. Esta bacteria es anaeróbica facultativa, y esta característica pudo favorecer su supervivencia en ambos sistemas. Aunque el amonio presente en las excretas líquidas tiene un efecto bactericida, los cocos gram positivos dada su menor permeabilidad celular son menos susceptibles a éste que otras bacterias (26). Según Poudel et al (27), solo es posible eliminar la población de $S$. aureus después de un TRH de 10 días, y en este estudio se refuerza dicho planteamiento, debido a que el TRH de mayor duración fue de 6.8 días.

En cuanto a mohos y levaduras, no hubo persistencia ni en los tanques estercoleros, ni en los biodigestores. Existen pocos datos sobre los recuentos de levaduras y mohos en los estiércoles animales (4). Se han encontrado mayor cantidad de bacterias que de hongos en porcinaza tratada por digestión anaerobia, debido a que las bacterias tienen un rol más importante en la conversión de residuos orgánicos durante este proceso (28). El presente estudio demuestra la eficiencia de los sistemas de tratamiento de porcinaza líquida para remover estos agentes, dada su susceptibilidad al amonio y sustancias húmicas (28).

Por su parte, se encontró persistencia de Salmonella spp., en los biodigestores, más no en los tanques estercoleros. Bajo las condiciones de este estudio, este agente no mostró persistencia en dichos tanques; las posibles razones de esta eliminación, podrían ser las altas concentraciones de amonio (26), y presencia de flora autóctona que compite directamente por el sustrato (9). Otros autores han encontrado persistencia de Salmonella spp. en porcinaza tratada por biodigestores anaerobios después de 30 a 40 días, aunque no siempre las cepas eran patogénicas (6). Aumentos de los TRH benefician la inactivación de Salmonella spp. (13). En este estudio, los bajos tiempos de retención probablemente no permitieron la remoción completa de Salmonella spp., en los efluentes de los biodigestores. Quizá las variaciones encontradas entre los autores consultados y los resultados de este estudio, se podrían explicar por la técnica de ausencia o presencia que se utilizó, donde se evaluaron $25 \mathrm{~g}$ de muestra que permite detectar muy bajas poblaciones 
Meanwhile, Salmonella spp. was found to be persistent in biodigesters but not in manure tanks. Under the conditions of this study, this agent did not show persistence in the tanks. Possible reasons for this elimination could be high concentrations of ammonium (26) and the presence of native flora which directly competes due to the substrate (9). Other authors have found Salmonella spp. to persist in pig manure treated in anaerobic biodigesters even after 3040 days, although the strains were not always pathogenic (6). Increased HRT contributes to inactivating Salmonella spp. (13). In this study, short retention times probably did not allow the complete removal of Salmonella spp., in biodigester effluent. Perhaps variations found among the authors consulted and the results of this study could be explained by the absence or presence technique, where $25 \mathrm{~g}$ of sample was used to detect very low amounts of Salmonella spp., which would depend on the prevalence of the disease on each farm.

Clostridium sulfite reducers persist in manure tanks and biodigesters. This can be explained because it is an anaerobic spore form that can resist and multiply in low redox potentials, and it is therefore likely to survive the anaerobic digestion of manure (3). The persistence of this bacteria has been reported in psychrophilic anaerobic digestion processes of pig manure using sequential batch reactors (12).

Total coliforms and $E$. coli persisted both in manure tanks and biodigesters. Son et al (26) added $2 \%$ urea (ammonium) in manure tanks and after two weeks obtained $<5 \mathrm{CFU} / \mathrm{g}$ values for total coliforms and $E$. coli due to the $\mathrm{pH}$ increase. Unlike this study, the manure tanks received no treatment. It can clearly be seen that retention times required for complete elimination of coliforms within a biodigester are greater than those in this study (maximum 6.8 days).

Anaerobic digestion has been highly efficient in removing $E$. coli with a HRT of 25 days (13). In this study $E$. coli persisted on all farms at all times; this can be explained by the short storage time. A difficulty that may arise when evaluating continuous flow anaerobic digesters is the lack of correlation between HRT and the reduction of pathogens such as $E$. coli, since a certain amount of pig manure passes quickly through the biodigester, since these biodigesters are continuously fed fresh pig manure. Low retention times could not only lead to increased survival of the pathogenic but also to increased problems with odors of effluents (29). Adding urea (ammonium) or increasing retention times de Salmonella spp., lo cual va a depender de la prevalencia de la enfermedad en cada granja.

Clostridium sulfito reductores persistieron en los tanques estercoleros, y en los biodigestores. Esto puede explicarse porque son anaerobios formadores de esporas que pueden soportar y multiplicarse en potenciales de oxido-reducción bajos, por lo tanto, es probable que logren sobrevivir a la digestión anaerobia del estiércol (3). La persistencia de estas bacterias ha sido reportada en procesos de digestión anaeróbica psicrófila de porcinaza usando reactores secuenciales discontinuos (12).

Los coliformes totales y E.coli persistieron tanto en los tanques estercoleros como en los biodigestores. Son et al (26), adicionaron $2 \%$ de urea (amonio) a estercoleros y después de dos semanas obtuvieron valores $<5 \mathrm{UFC} / \mathrm{g}$ para coliformes totales y E.coli, por efecto del aumento del $\mathrm{pH}$. A diferencia de este estudio, los tanques estercoleros no recibieron ningún tratamiento. Puede observarse claramente que los tiempos de retención requeridos para la completa eliminación de la población de coliformes dentro de un biodigestor, son mayores que los de este estudio (máximo 6.8 días).

La digestión anaerobia ha exhibido alta eficiencia en la remoción de $E$. coli con TRH de 25 días (13). En este trabajo E. coli persistió en todas las granjas y en todo momento; explicado por el poco tiempo de almacenamiento. Una dificultad que puede surgir de la evaluación de biodigestores anaerobios de flujo continuo, es la falta de correlación entre el TRH y la reducción de patógenos como $E$. coli, debido a que una cierta cantidad de porcinaza pasa más rápido a través del biodigestor, dado que son biodigestores que continuamente son alimentados con porcinaza fresca. Un bajo tiempo de retención no sólo podría conducir a un aumento de la supervivencia de patógenos, sino también a incrementar los problemas con los malos olores de los efluentes (29). La adición de urea (amonio) o la prolongación del tiempo de retención son alternativas a evaluar en trabajos posteriores para minimizar el riesgo que representan estos microorganismos.

L. monocytogenes solo fue observada y persistió en una muestra en estercolero. Es posible que la combinación de un ambiente pobre en materia orgánica, rico en sal y con un $\mathrm{pH}$ alcalino, favorezca la supervivencia de este patógeno reconocido por soportar condiciones extremas de estrés (30). 
are alternatives to be evaluated in further studies in order to minimize the risk posed by these microorganisms.

L. monocytogenes was observed and persisted in one sample manure tank. It is possible that the combination of an environment poor in organic matter, rich in salt, and an alkaline $\mathrm{pH}$ favored the survival of this pathogen that is known to withstand extreme stress conditions (30).

As for parasites in the manure, persistence of $B$. coli or Strongylid is not observed, while coccidia persisted. As Son et al (26) observed, conditions that favor high ammonium concentrations may inactivate parasites. As for Strongyloides spp., persistence was observed but it is known that free-living states of Strongyloides spp., have a short duration and are negatively affected by adverse environmental conditions. The persistence of coccidia may be explained by the liquid medium which favors the development of parasites and additionally due to its ability to form oocytes that are highly resistant to unfavorable environmental conditions. In nature, oocytes probably survive for weeks or months. In the case of biodigesters, persistence of Strongylid and Strongyloides spp., was not found, but coccidian was found. Biological considerations of these parasites described in pig manure tanks apply similarly to biodigesters. Cañon-Franco et al (24) evaluated the efficiency of anaerobic digesters in eliminating parasites after 15 days of HRT, finding that the time is insufficient for complete removal of Isospora suis oocytes and different species of Eimeria such as Strongyloides ransomi eggs. In this study the results are similar in terms of the persistence of coccidia, but not in terms of Strongyloides spp. The persistence of coccidia on both routes could be explained by its cystic nature, which is very resistant to adverse environmental conditions. In this study, retention times were low compared to reports in literature, however, it was possible to eliminate some of the parasites. Very short HRT does not allow adequate removal of pathogens, particularly of parasite eggs, which have greater resistance to environmental stress as compared to viruses and bacteria (8).

In this study the results for manure tanks show that PCV2, molds, yeasts, Salmonella spp., B. coli and Strongylid did not persist, while L. intracellularis, aerobic mesophilic bacteria, S. aureus, Clostridium sulfite reducers, total coliforms, E. coli, L. monocytogenes, Strongyloides spp., and coccidia did persist. On the other hand, in the biodigesters it can be seen that PCV2, yeast, strongylid, $B$. coli and Strongyloides spp. did not persist,
En cuanto a parásitos en el estercolero, no se observó persistencia de $B$. coli ni de estrongilidos, mientras las coccidias si persistieron. Como lo menciona Son et al (26), condiciones que favorezcan altas concentraciones de amonio pueden inactivar parásitos. En cuanto a los Strongyloides spp., se observó persistencia, al respecto, se sabe que los estados de vida libre de Strongyloides spp., tienen corta duración y son afectadas negativamente por las condiciones adversas del ambiente. La persistencia de coccidias puede explicarse por trabajarse en un medio líquido que es favorable para el desarrollo de estos parásitos y adicionalmente por su capacidad de formar ooquistes muy resistentes a condiciones ambientales desfavorables. En la naturaleza, los ooquistes probablemente sobreviven por semanas o meses. En el caso de los biodigestores, no se encontró persistencia de estrongilidos ni Strongyloides spp., pero sí de coccidias. Las consideraciones biológicas de estos parásitos descritas en los tanques estercoleros, aplican de la misma manera para los biodigestores. Cañon-Franco et al (24), evaluaron la eficiencia de biodigestores anaerobios en la eliminación de parásitos después de 15 días de $\mathrm{TRH}$, encontrando que ese tiempo es insuficiente para la eliminación completa de ooquistes de Isospora suis y diferentes especies de Eimeria, así como huevos de Strongyloides ransomi. En este trabajo los resultados son semejantes en cuanto a la persistencia de coccidias, pero no en cuanto a Strongyloides spp. La persistencia de coccidias en ambas rutas podría explicarse por su naturaleza quística, las cuales son formas muy resistentes a las condiciones adversas del ambiente. En este trabajo los tiempos de retención fueron bajos respecto a los reportes encontrados en la literatura, sin embargo, fue posible la eliminación de algunos de los parásitos considerados. TRH muy cortos no permiten una adecuada eliminación de patógenos, en particular de huevos de parásitos, los cuales tienen una mayor resistencia al estrés ambiental comparado con virus y bacterias (8).

En el presente trabajo los resultados en tanques estercoleros muestran que PCV2, mohos, levaduras, Salmonella spp., B. coli y estrongilidos no persistieron; mientras que $L$. intracellularis, mesófilos aerobios, S.aureus, Clostridium sulfito reductores, coliformes totales, E. coli, L. monocytogenes, Strongyloides spp., y coccidias si persistieron. De otro lado, en los biodigestores se aprecia que PCV2, levaduras, estrongilidos, B. coli y Strongyloides spp., no persistieron; mientras que $L$. intracellularis, mesófilos aerobios, S.aureus, Clostridium sulfito reductores, coliformes totales, E. coli, Salmonella spp., L. monocytogenes, y coccidias si lo hicieron. 
while L. intracellularis, aerobic mesophilic bacteria, $S$. aureus, Clostridium sulfite reducers, total coliforms, E. coli, Salmonella spp., L. monocytogenes, and coccidia did.

Under the conditions of this study it was not possible to guarantee the safety of pig manure since some pathogens managed to persist in spite of various treatments. However, both manure tanks and biodigesters showed variations in persistence, indicating that they work as systems to transform pig manure to remove some pathogens, though it is necessary to increase storage times to improve efficiency, which, for the biodigesters, can be improved by increasing the size or by using less wash water in the facilities.

It is important to consider for further studies if the eggs or larvae of parasites, as well as viruses and bacteria, found to be persistent are viable or not. In the case of Salmonella spp., it is necessary to verify whether they are pathogenic or nonpathogenic strains, and for $E$. coli differentiate the type of adhesins, since these are specific to each species; for pigs K88, K99 for cattle.
Bajo las condiciones de este estudio no es posible garantizar la inocuidad de la porcinaza puesto que algunos agentes patógenos lograron persistir a los diversos tratamientos. Sin embargo, tanto en estercoleros como biodigestores se observó variación en la persistencia, indicando que funcionan como sistemas de transformación de la porcinaza para la remoción de algunos patógenos, siendo necesario aumentar los tiempos de almacenamiento si se quiere mejorar su eficiencia, el cual, en el caso de los biodigestores, puede aumentarse por una ampliación en su tamaño, o por la utilización de menor cantidad de agua de lavado en las instalaciones.

Es importante considerar para estudios posteriores, si los huevos o larvas de parásitos, así como los virus y bacterias en los que se encontró persistencia son viables o no. En el caso de Salmonella spp., verificar si se trata de cepas patógenas o apatógenas y para $E$. coli diferenciar el tipo de adhesinas pues estas son específicas para cada especie K88 para porcinos, K99 para bovinos.

\section{REFERENCES}

1. McGlone JJ. The future of pork production in the world: towards sustainable, welfarepositive systems. Animals 2013; 3(2):401-15.

2. Asociación Colombiana de Porcicultores. Análisis de coyuntura del sector porcícola año 2013. Porcicul Colomb 2014; 3(2):16-30.

3. Pell AN. Manure and microbes: public and animal health problem? J Dairy Sci 1997; 80(10):2673-81.

4. McCarthy G, Lawlor PG, Gutierrez M, Gardiner $\mathrm{GE}$. Assessing the biosafety risks of pig manure for use as a feedstock for composting. Sci Total Environ 2013; 463-464:712-9.

5. Goss M, Richards C. Development of a riskbased index for source water protection planning, which supports the reduction of pathogens from agricultural activity entering water resources. J Environ Manage 2008; $87(4): 623-32$.

6. Fongaro $G$, Viancelli $A$, Magri ME, Elmahdy EM, Biesus LL, Kich JD, et al. Utility of specific biomarkers to assess safety of swine manure for biofertilizing purposes. Sci Total Environ 2014; 479-480:277-83.
7. Rivas O, Vargas F, Watson G. Biodigestores: factores químicos, físicos y biológicos relacionados con su productividad. Tecnol March 2010; 23(1):39-46.

8. Huong LQ, Madsen $\mathrm{H}$, Anh le $\mathrm{X}$, Ngoc PT, Dalsgaard A. Hygienic aspects of livestock manure management and biogas systems operated by small-scale pig farmers in Vietnam. Sci Total Environ 2014; 470-471:53-7.

9. Smith SR, Lang NL, Cheung KH, Spanoudaki $\mathrm{K}$. Factors controlling pathogen destruction during anaerobic digestion of biowastes. Waste Manag 2005; 25(4):417-25.

10. Appels L, Baeyens J, Degrève J, Dewil R. Principles and potential of the anaerobic digestion of waste-activated sludge. Prog Energy Combust Sci 2008; 34(6):755-81.

11. Olugasa TT, Odesola IF, Oyewola MO. Energy production from biogas: A conceptual review for use in Nigeria. Renew Sust Energ Rev 2014; 32:770-6.

12. Masse D, Gilbert Y, Topp E. Pathogen removal in farm-scale psychrophilic anaerobic digesters processing swine manure. Bioresour. Technol $2011 ; 102(2): 641-6$. 
13. Chen $Y$, Fu B, Wang $Y$, Jiang Q, Liu H. Reactor performance and bacterial pathogen removal in response to sludge retention time in a mesophilic anaerobic digester treating sewage sludge. Bioresour Technol. 2012; 106:20-6.

14. Estrada- Alvarez J, Gómez-Londoño G, Jaramillo-Jimenez A. Efecto del biodigestor plástico de flujo continuo en el tratamiento de aguas residuales de establos bovinos. Vet Zootec 2008; 2(2):9-20.

15. Instituto Colombiano de Normas Técnicas y Certificación (ICONTEC). Norma Técnica Colombiana NTC-ISO 5667-1. Gestión ambiental, Calidad del agua, Muestreo, Directrices para el diseño de programas de muestreo. Bogotá, Colombia: ICONTEC; 1995.

16. Huang C, Hung JJ, Wu CY, Chien MS. Multiplex PCR for rapid detection of Pseudorabies Virus, Porcine Parvovirus and Porcine Circoviruses. Vet Microbiol 2004;101(3):209-14.

17. Urbaniak K, Markowska-Daniel I. Application of PCR based on apxIVA gene for rapid detection of Actinobacillus pleuropneumoniae. Bull Vet Inst Pulawy 2011; 55(4):609-12.

18. Asawakarn S, Watanaphansak S, Asawakarn T. Report of Lawsonia intracellularis Infection in Dogs by Polymerase Chain Reaction. Thai J Vet Med 2012; 42(4):523-26.

19. Lurchachaiwong W, Payungporn S, Srisatidnarakul U, Mungkundar C, Theamboonlers A, Poovorawan Y. Rapid detection and strain identification of porcine reproductive and respiratory syndrome virus (PRRSV) by real-time RT-PCR. Lett Appl Microbiol 2008; 46(1):55-60.

20. Instituto Colombiano Agropecuario (ICA). Directiva DIP-30-100-003. Directivas técnicas de alimentos para animales y sales mineralizadas. Alimentos para animales, parámetros microbiológicos. Instituto Colombiano Agropecuario, Ministerio de Agricultura y Desarrollo Rural. [en línea] 1999. [acceso julio de 2014] URL disponible en: http://www.ica.gov.co/getdoc/7d27ee5ecfe 4- 4 7a 2-868e-7c53f 4 e 49473/ Directivastecnicasalimentosanimales.aspx

21. Instituto Nacional de Salud, Ministerio de la Protección Social. Evaluación de riesgos de Staphylococcus aureus enterotoxigénico en alimentos preparados no industriales en Colombia. Imprenta Nacional de Colombia. [en línea] 2011. [acceso julio de 2014] URL disponible en: http://www.ins.gov.co/lineasde-accion/investigacion/ueria/Publicaciones/ ER\%20STAPHYLOCOCCUS.pdf
22. Gilbert RJ, de Louvois J, Donovan T, Little C, Nye $K$, Ribeiro $C D$, et al. Guidelines for the microbiological quality of some ready-to-eat foods sampled at the point of sale. Commun Dis Public Health 2000; 3(3):163-7.

23. Turner $\mathrm{C}$, Burton $\mathrm{CH}$. The inactivation of viruses in pig slurries: a review. Bioresour. Technol 1997; 61(1):9-20.

24. Cañon-Franco WA, Henao-Agudelo RA, PérezBedoya JL. Recovery of gastrointestinal swine parasites in anaerobic biodigester systems. Rev Bras Parasitol Vet 2012; 21(3):249-53.

25. Park K, Thompson A, Marinier M, Clark K, Wagner-Riddle $C$. Greenhouse gas emissions from stored liquid swine manure in a cold climate. Atmos Environ 2006; 40(4): 618-27.

26. Son TTD, Truong DV, Madsen H, Dalsgaard A. Survival of faecal indicator bacteria in treated pig manure stored in clay-covered heaps in Vietnam. Vet Microbiol 2011; 152(3-4):374-8.

27. Poudel RC, Joshi DR, Dhakal NR, Karki AB. Anaerobic digestion of sewage sludge mixture for the reduction of indicator and pathogenic microorganisms. Sci World 2010; 8(8):47-50.

28. Cao Y, Chang Z, Wang J, Ma Y, Fu G. The fate of antagonistic microorganisms and antimicrobial substances during anaerobic digestion of pig and dairy manure. Bioresour. Technol 2013; 136:664-71.

29. Huong LQ, Forslund A, Madsen H, Dalsgaard A. Survival of Salmonella spp. and fecal indicator bacteria in Vietnamese biogas digesters receiving pig slurry. Int J Hyg Environ Health 2014; 217(7):785-95.

30. Pourcher AM, Ziebal C, Kervarrec M, Bioteau T, Dabert P. Sanitary status of 44 hog manures in Brittany: comparison of the effectiveness of manure treatments based on the levels of indicator bacteria and two pathogenic bacteria. J Agr Sci Tech 2012; 3(A2):303-13. 